\title{
Lyapunov-based Friction Compensation for Accurate Positioning of a Hydraulic Actuator
}

\author{
P. Sekhavat, Q. Wu and N. Sepehri* \\ Department of Mechanical and Industrial Engineering \\ The University of Manitoba \\ Winnipeg, Manitoba, Canada R3T 5V6
}

\begin{abstract}
A Lyapunov-based discontinuous friction compensation technique is developed for the position regulation of a hydraulic actuator. The control scheme is capable of asymptotic position regulation with no steadystate error despite friction effects. Although, no knowledge of actuator friction, servovalve dynamics, or hydraulic parameters is required for control action, stability and effectiveness of the control scheme considering hydraulic nonlinearities, servovalve dynamics, and realistic friction model is verified both analytically and experimentally. Due to the discontinuity of the control law and the friction model, the control system is nonsmooth. Therefore, existence, continuation and uniqueness of the Filippov's solution are, first, proven using Filippov's solution theories. The extension of LaSalle's invariance principle to nonsmooth systems is then employed to prove the asymptotic convergence of the system trajectories to the equilibria. Experimental results verify the effectiveness of the proposed controller in counteracting frictional effects and asymptotic convergence of the system to the desired position with no steady state error.
\end{abstract}

\section{Introduction}

Hydraulic systems have been widely used in many industrial positioning applications such as assembly tasks, heavy-duty manipulators, and material testing equipments. Unlike their electrical counterparts, hydraulic systems exhibit highly nonlinear characteristics [1]. Previous studies on hydraulic systems have demonstrated that nonlinear control schemes can achieve a better performance than the conventional linear controllers [2,3]. Most of recent studies within the context of hydraulically actuated position control are, therefore, based on the advanced nonlinear control theories such as feedback linearization [1], adaptive control $[2,4-6]$, variable structure control [7], and direct Lyapunov-based control [8,9]. However, they did not consider dry friction that is a major disturbance in hydraulic cylinders and compensating it could improve the positioning performance [3]. In fact, feedback control for

This work was supported by the Natural Sciences and Engineering Research Council of Canada (NSERC) and the Institute for Robotics and Intelligent Systems (IRIS) Network Centre of Excellence.

* Corresponding author: nariman@cc.umanitoba.ca precise positioning has been a challenging task due to the presence of actuator friction.

Friction effects on hydraulic systems are of more importance compared to electrical systems. Degrading effects of friction (especially in small motions at low speeds) dominates the behavior of position control systems, making precise position control difficult to achieve. This is due to the fact that hydraulic actuators operate under high supply pressures and tight sealing is required to prevent them from internal leakage. Typical errors caused by friction include steady-state errors in position regulation (caused by static friction), tracking lags (caused by viscous friction) [10], limit cycles, and undesired stick-slip motion. Friction effects may even cause instability during position or force feedback control [6]. Compensating friction in hydraulic systems is, however, difficult since the control input drives the derivative of the actuator force and therefore one cannot directly cancel friction term as is commonly done in electric motors.

Armstrong-Helouvry et al. [11] conducted an extensive survey on existing friction compensation techniques divided into two major non-model based and model based compensation categories. Since the physical nature of friction is such that it can be rarely determined a priori with the required accuracy and even if estimated it often changes with time, most of the model-based methods entail on-line estimation of the friction using observers [10,11]. An alternative approach has been employing Lyapunov's direct method to develop a nonlinear discontinuous compensation technique that stabilizes the system at the desired position [12]. The present study follows the latter idea to enable exact position regulation in hydraulic actuators in spite of actuator friction.

Within the context of friction compensation in hydraulic systems, Lischinsky et al [6] used a nonlinear PI-type controller in the inner torque loop of an outer position control of a hydraulic manipulator. Neglecting the valve dynamics, they provided the stability proof of the position control system. Tafazoli et al. [5] established an adaptive friction compensation technique by combining observerbased friction estimation with an acceleration feedback control. They did not include the valve dynamics and the stability analysis was somewhat less rigorous. Yao et al. [2] 
included the dynamics of servovalve and proposed a discontinuous adaptive robust controller supported by a rigorous stability proof for position tracking. In case of position regulation, however, complete stability analysis was not provided; nor they provided a solution analysis of the nonsmooth system under study. Sohl and Bobrow [9] obtained a nonlinear control law using the Lyapunov stability analysis. Their control law required knowledge of some system parameters (valve coefficients, fluid bulk modulus, Coulomb friction) whose values may change during the position control process.

In the present study we design and experimentally validate a Lyapunov-based nonlinear friction compensation technique for the asymptotic position regulation of a hydraulic actuator with no steady-state error in the presence of actuator friction. Although, no knowledge of the friction effects, servovalve dynamics, or hydraulic parameters is required for control action, stability and effectiveness of the control scheme considering hydraulic nonlinearities, servovalve dynamics, and the realistic friction model is verified both analytically and experimentally. Due to the discontinuous nature of the actuator friction model and the proposed control law, the control system is nonsmooth. In this study, the existence, continuation and uniqueness of Filippov's solution $[13,14]$ of the hydraulic position control system in the presence of friction are investigated. The extension of Lyapunov stability theory to nonsmooth systems $[15,16]$ is then employed to guarantee the global asymptotic convergence of the entire system's states towards the equilibria. Experimental results show the effectiveness and applicability of the proposed control.

\section{Dynamic Model of the System}

\subsection{Model of Hydraulic System}

Figure 1 shows the schematic diagram of the double-ended hydraulic actuation system under study. The equation of motion of the actuator is described by the following second-order equation

$m \ddot{x}=A P_{L}-F_{f}$

where $x$ is the piston displacement and $F_{f}$ is the friction force. Parameters $m$ and $A$ are the mass of actuator's moving parts and piston area, respectively. $P_{L}=P_{i}-P_{o}$ is the load pressure. For valves with rectangular matched and symmetric orifice areas, $P_{L}$ changes with time according to the following relation (neglecting leakages) [17]:

$\dot{P}_{L}=\frac{1}{C}\left(-A \dot{x}+\frac{c_{d} w}{\sqrt{\rho}} x_{s p} \sqrt{P_{s}-\operatorname{sign}\left(x_{x p}\right) P_{L}}\right)$

where $\dot{x}$ is the actuator velocity, $w$ is the orifice area gradient, $\rho$ is the hydraulic fluid density, $P_{s}$ is the pump pressure, and $c_{d}$ is the orifice coefficient of discharge. $x_{s p}$ is the spool displacement and $C=V_{t} / 4 \beta$ is the hydraulic compliance where $V_{t}$ is the total actuator volume and $\beta$ is the effective bulk modulus of the system. The function $\operatorname{sign}\left(x_{s p}\right)$ in (2) is defined as:

$\operatorname{sign}\left(x_{s p}\right)=\left\{\begin{array}{cc}x_{s p} /\left|x_{s p}\right| & ; \quad x_{s p} \neq 0 \\ 0 & ; \quad x_{s p}=0\end{array}\right.$

The dynamics between the spool displacement, $x_{s p}$, and the input voltage, $u$, is modeled as a first-order system [18]:

$\dot{x}_{s p}=-\frac{1}{\tau} x_{s p}+\frac{k_{s p}}{\tau} u$

$k_{s p}$ and $\tau$ are valve gain and time constant, respectively.

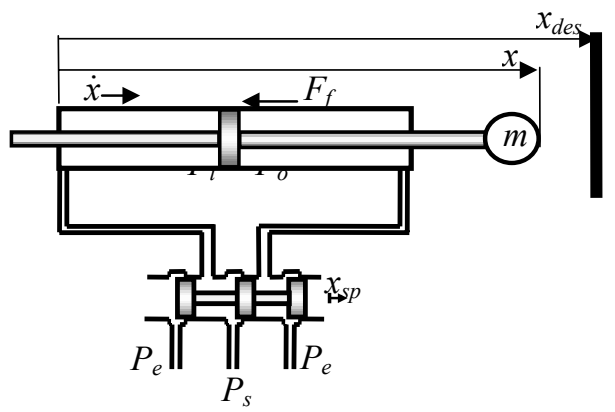

Fig. 1 Schematic of the system configuration.

\subsection{Friction Model}

It is well known that the major components of friction are Coulomb force, viscous force, and Stribeck effects. Experiments have confirmed Tustin's discontinuous model that includes the above components in the friction model [10]. The model is widely used in many general [11] as well as hydraulic [5] position and force control systems:

$F_{f}=\left\lfloor F_{C}+\left(F_{S}-F_{C}\right) e^{-\left(\dot{x} / \dot{x}_{s}\right)^{2}}\right\rfloor \operatorname{sgn}(\dot{x})+d \dot{x}$ where $F_{\mathrm{C}}$ is the Coulomb friction, $F_{\mathrm{S}}$ is the stiction force (the force needed to start the motion), $\dot{x}_{s}$ is a threshold velocity where the downward bend in friction appears after the stiction (breakaway) force is surmounted, and $d$ is the viscous friction coefficient. At rest, the static friction $\left(F_{S} \operatorname{sgn}(0)\right)$ is opposite to the applied force and can acquire any value in the range of $\left[-F_{S}, F_{S}\right]$. This opposing static friction increases with the increase in the net external force until it reaches the breakaway force, $F_{S}$, where the piston starts to slide and the friction drops due to Stribeck effect. The function $\operatorname{sgn}(\dot{x})$ is, thus, defined as:

$$
\left\{\begin{array}{lll}
\operatorname{sgn}(\dot{x})=\{\dot{x} /|\dot{x}|\} & : & \dot{x} \neq 0 \\
\operatorname{sgn}(\dot{x}) \in[-1,1] & : & \dot{x}=0
\end{array}\right.
$$

\section{Controller Design}

In designing the friction compensation position control scheme, the goal is to have the piston reach the desired position, $x_{d e s}$, with no steady state error. Using the Lyapunov's direct method (detailed in Section 5), the following position control law is proposed:

$u=-\left(K_{p}\left|e_{3}\right| \operatorname{sign}\left(e_{4}\right)+K_{x} e_{1}\right) \sqrt{P_{s}-\operatorname{sign}\left(e_{4}\right) e_{3}}$

where $K_{p}$ and $K_{x}$ are positive constant gains. 
Measurements of the ram position, hydraulic line pressures, supply pressure and the knowledge about the direction of the valve spool displacement are the requirements for the implementation of the above control algorithm. Existence of the pressure feedback in the control law is recommended by researchers for damping out the resonance of hydraulic cylinder and achieving a higher bandwidth [5].

In order to constitute the state space model of the system, the vector of error states are defined as $\mathbf{e}=\left(e_{1}, e_{2}, e_{3}, e_{4}\right)^{T}$ where

$e_{1}=x-x_{d e s} \quad, \quad e_{2}=\dot{x} \quad, \quad e_{3}=P_{L} \quad, \quad e_{4}=x_{s p}$

Combining equations (1)-(8) results in the following errorspace model of the system:

$$
\left\{\begin{array}{l}
\dot{e}_{1}=e_{2} \\
\dot{e}_{2}=\frac{A}{m} e_{3}-\frac{\left[F_{C}+\left(F_{S}-F_{C}\right) e^{-\left(e_{2} / \dot{x}_{s}\right)^{2}}\right] \operatorname{sgn}\left(e_{2}\right)+d e_{2}}{m} \\
\dot{e}_{3}=\frac{1}{C}\left(-A e_{2}+\frac{c_{d} w}{\sqrt{\rho}} e_{4} \sqrt{P_{s}-\operatorname{sign}\left(e_{4}\right) e_{3}}\right) \\
\dot{e}_{4}=-\frac{1}{\tau} e_{4}-\frac{k_{s p}}{\tau}\left(K_{p}\left|e_{3}\right| \operatorname{sign}\left(e_{4}\right)+K_{x} e_{1}\right) \sqrt{P_{s}-\operatorname{sign}\left(e_{4}\right) e_{3}}
\end{array}\right.
$$

The above model does not incorporate the dead-zone nonlinearities caused by possible spool valve overlap. Leakage flow across the actuator's piston is also neglected since it inherently has stabilizing effect by providing damping for hydraulic resonant mode [1]. For the above double-rod cylinder, directional nonlinearity is not an issue and the servovalve saturation nonlinearity can also be neglected if the valve is never allowed to saturate.

The equilibria of the above system are obtained by equating the right-hand side of (9) to zero:

$$
\left\{\begin{array}{l}
e_{2 s s}=0 \\
e_{3 s s}=\frac{F_{S} \operatorname{sgn}(0)}{A} \\
e_{4 s s}=0 \\
e_{1 s s}=0
\end{array}\right.
$$

where $F_{S} \operatorname{sgn}(0) \in\left[-F_{S}, F_{S}\right]$ represents the static friction during the steady state and is equal and opposite to the external applied force. Thus, the equilibrium point of the system is every $\mathbf{e}_{e q}=\left(0,0, e_{3 s s}, 0\right)^{T}$ where

$e_{3 s s}=\frac{F_{S} \operatorname{sgn}(0)}{A} \in\left[-\frac{F_{S}}{A}, \frac{F_{S}}{A}\right]$

Therefore, despite friction effects, the equilibria of the system shown in (9) are always where the piston position approaches the desired position asymptotically.

Inclusion of the discontinuous friction model and the discontinuous nature of the control law in the analysis results in a nonsmooth system. Here, Filippov's solution concept is used to investigate the solution of discontinuous control systems under study.

\section{Solution Analysis}

With reference to (9), the discontinuity surface of the system is one of the following three surfaces:

Surface $1 \quad S_{1}^{3}:=\left\{\mathbf{e}: e_{2}=0 \& e_{4} \neq 0\right\}$

Surface $2 S_{2}^{3}:=\left\{\mathbf{e}: e_{2} \neq 0 \& e_{4}=0\right\}$

Surface $3 S_{1}^{2}:=\left\{\mathbf{e}: e_{2}=0 \& e_{4}=0\right\}$

The subscript and superscript denote the dimension and the number of the discontinuity surface, respectively. The surface $S_{1}^{2}$ is the intersection of surfaces $S_{1}^{3}$ and $S_{2}^{3}$.

\subsection{Existence and continuation of Filippov's solution}

Let region $\Omega=R^{4} \times R$ and let $D$ be an arbitrary compact set in $\Omega$. The right-hand sides of equations (9) are defined everywhere in $\Omega$, and are bounded by $B(t)$. Let $B(t)=L$, which is obviously integrable on $D$. Furthermore, each term of the right-hand sides of (9) is measurable. Thus, the righthand sides of equations (9) satisfy condition B of the Filippov's solution theory [13] and we have the local existence of a solution which is continuable on $\left[t_{0}, t_{f}\right]$.

\subsection{Uniqueness of Filippov's solution}

The vector-valued function of the right-hand sides of equations (9) is continuous up to the discontinuity surfaces and the discontinuity surfaces are smooth and independent of time $t$. Therefore, conditions A, B and C of Filippov's solution theory [14] are satisfied. Next, the analysis of the uniqueness of Filippov's solution must be carried out for each discontinuity surface.

We first study the uniqueness of Filippov's solution for the discontinuity surface $S_{1}^{3} \cdot S_{1}^{3}$ divides the solution region into two parts: $\Omega^{+}:=\left\{\mathbf{e}: e_{2}>0\right\}$ and $\Omega^{-}:=\left\{\mathbf{e}: e_{2}<0\right\}$. The normal to this surface, denoted by $\mathbf{N}_{\mathbf{s}_{1}^{3}}$, is:

$\mathbf{N}_{\mathbf{s}_{1}^{3}}=\left\{\begin{array}{llll}\frac{\partial S_{1}^{3}}{\partial e_{1}} & \frac{\partial S_{1}^{3}}{\partial e_{2}} & \frac{\partial S_{1}^{3}}{\partial e_{3}} & \frac{\partial S_{1}^{3}}{\partial e_{4}}\end{array}\right\}^{T}=\left\{\begin{array}{llll}0 & 1 & 0 & 0\end{array}\right\}^{T}$

Defining the vector functions $\mathbf{f}^{+}$and $\mathbf{f}^{-}$as the limiting values of the right-hand sides of the state- space equations (9) in regions $\Omega^{+}$and $\Omega^{-}$, the projections of $\mathbf{f}^{+}$and $\mathbf{f}$ along the normal to the discontinuity surface, $S_{1}^{3}$, are:

$\mathbf{f}_{\mathbf{N}_{1}^{3}}^{+}=\mathbf{f}^{+} \cdot \mathbf{N}_{\mathbf{s}_{1}^{3}}=\frac{A}{m} e_{3}-\frac{F_{S}}{m}$

$\mathbf{f}_{\mathbf{N}_{1}^{3}}^{-}=\mathbf{f}^{-} \cdot \mathbf{N}_{\mathbf{s}_{1}^{3}}=\frac{A}{m} e_{3}+\frac{F_{S}}{m}$

Therefore: $\quad \mathbf{f}_{\mathbf{N}_{1}^{3}}^{+}-\mathbf{f}_{\mathbf{N}_{1}^{3}}^{-}=-2 \frac{F_{S}}{m}<0$

Thus, according to Theorem 14 of Filippov [13], the uniqueness of the Filippov's solution is guaranteed. The uniqueness analysis for $S_{2}^{3}$ can be done in a similar way. 
Uniqueness analysis of $S_{1}^{2}$ requires heavier mathematical machinery.

\section{$5 \quad$ Stability Analysis}

Although Lyapunov's second method was originally developed for smooth nonlinear systems, its extension to nonsmooth systems based on Filippov's solution theory $[15,16]$ has provided the theoretical foundation for proper stability analysis of non-smooth systems. The stability analysis of the system shown in (9) is conducted using the extension method introduced in [16] and deriving a smooth regular function for the system under study.

Let $V$ be the positive regular function introduced for the nonsmooth system given in (9):

$V=\frac{1}{2}\left(\frac{A e_{1}}{C}+e_{3}\right)^{2}+\frac{\varepsilon m}{2 C} e_{2}^{2}+\frac{\varepsilon}{2} e_{3}^{2}+\frac{\tau c_{d} w A}{2 k_{s p} K_{x} \sqrt{\rho} C^{2}} e_{4}^{2}$

where $\varepsilon$ is an arbitrary small positive constant. The derivative of the above function is

$$
\begin{aligned}
& \dot{V}=-\frac{c_{d} w A}{k_{s p} K_{x} \sqrt{\rho} C^{2}} e_{4}^{2}-\frac{\varepsilon\left(F_{C}+\left(F_{S}-F_{C}\right) e^{-\left(e_{2} / \dot{x}_{s}\right)^{2}}\right)}{C}\left|e_{2}\right|-\frac{\varepsilon d}{C} e_{2}^{2} \\
& -\frac{c_{d} w}{C \sqrt{\rho}}\left(\frac{A K_{p}}{C K_{x}}\left|e_{3} e_{4}\right|-(\varepsilon+1) e_{3} e_{4}\right) \sqrt{P_{s}-\operatorname{sign}\left(e_{4}\right) e_{3}}
\end{aligned}
$$

According to (17), in order to have negative semi-definite $\dot{V}$, the following condition is imposed on the control gain ratio:

$$
\frac{K_{p}}{K_{x}}>\frac{(\varepsilon+1) C}{A}
$$

Not that since $0<\varepsilon<<1$, equation (18) implies that the lower limit on the control gain ratio is $C / A$.

Equations (17) and (18) denote that $\dot{V}$ is continuous and negative semi-definite throughout the solution region except for the discontinuity surfaces. On $S_{1}^{3}$ :

$\dot{V}\left(\mathbf{e} \in S_{1}^{3}\right) \in \operatorname{co}\left[\dot{V}^{S_{1}^{3^{+}}}, \dot{V}^{S_{1}^{3^{-}}}\right]$

where $\dot{V}^{S_{1}^{3^{+}}}$and $\dot{V}^{S_{1}^{3^{-}}}$are the limit values of $\dot{V}$ as a solution trajectory approaches $S_{1}^{3}$ from both sides:

$$
\begin{aligned}
& \dot{V}^{S_{1}^{3^{+}}}=\lim _{e_{4} \rightarrow 0^{+}} \dot{V}=-\frac{\varepsilon\left(F_{C}+\left(F_{S}-F_{C}\right) e^{-\left(e_{2} / \dot{x}_{s}\right)^{2}}\right)}{C}\left|e_{2}\right|-\frac{\varepsilon d}{C} e_{2}^{2} \\
& \dot{V}^{S_{1}^{3^{-}}}=\lim _{e_{4} \rightarrow 0^{-}} \dot{\dot{V}}=-\frac{\varepsilon\left(F_{C}+\left(F_{S}-F_{C}\right) e^{-\left(e_{2} / \dot{x}_{S}\right)^{2}}\right)}{C}\left|e_{2}\right|-\frac{\varepsilon d}{C} e_{2}^{2}
\end{aligned}
$$

Equations (20) imply that the convex set described in (19) only contains one negative element. Thus, on the discontinuity surface $S_{1}^{3}$ :

$\dot{V}\left(\mathbf{e} \in S_{1}^{3}\right)=-\frac{\varepsilon\left(F_{C}+\left(F_{S}-F_{C}\right) e^{-\left(e_{2} / \dot{x}_{s}\right)^{2}}\right)}{C}\left|e_{2}\right|-\frac{\varepsilon d}{C} e_{2}^{2}$

Similar proof can be derived for the derivative of the $V$ on
$S_{2}^{3}$ and $S_{1}^{2}$ - details are not presented for the sake of brevity. We can, thus, conclude that $\dot{V}$ is negative semidefinite in both contact and noncontact regions of motion as well as on the discontinuity surfaces. Therefore, according to the extended LaSalle's invariance principle to nonsmooth systems, every solution trajectory in $\Omega$ globally converges to the largest invariant set, $\mathbf{M}$, as $t \rightarrow \infty$. Next, we prove that the largest invariant set, $\mathbf{M}$, contains only the points that belong to the set $\mathbf{e}_{e q}=\left(0,0, e_{3 s s}, 0\right)^{T}$ with $e_{3 s s}$ defined in (11). This can be proven by contradiction. Let $\mathbf{R}$ be the set of all points within the solution region $\Omega$ where $\dot{V}=0$. With respect to (17), $\dot{V}=0$ requires that for all the points in $\mathbf{R}, e_{2}=0$ and $e_{4}=0$. Thus, both $\dot{e}_{2}$ and $\dot{e}_{4}$ are zero. Let $\mathbf{M}$ be the largest invariant set in $\mathbf{R}$ and contain a point where either $e_{1} \neq 0$ and/or $e_{3}$ is not equal to the value shown in (11). According to equations (9b) and (9d), this will result in either $\dot{e}_{2} \neq 0$ and/or $\dot{e}_{4} \neq 0$ which necessitates the solution trajectory to immediately move out of the set $\mathbf{R}$ and certainly set $\mathbf{M}$. But, this conclusion contradicts with the initial assumption that $\mathbf{M}$ is the largest invariant set in $\mathbf{R}$. Thus, $e_{1}$ can only be equal to zero and $e_{3}$ can only be equal to the value shown in (11). This discussion concludes that every solution trajectory in $\Omega$ will converge to the largest invariant set $\mathbf{M}$ containing only the equilibria. The control scheme is, thus, capable of asymptotic position regulation with no steady-state error despite friction effects.

\section{Experimental Verification}

Experiments were conducted on an electrohydraulic actuator following a desired position. The goal is to verify the theoretical conclusion drawn in Section 3 and show that the proposed control scheme in this paper can effectively counteract frictional effects and achieve the desire position asymptotically. The first set of experiments was performed with control gains chosen as $K_{p} / K_{x} \cong C / A$. Realistically, the exact values of $C$ and $A$ may not be available. Therefore, the second set of experiments was conducted to observe the performance of the control scheme if the control gain ratio is almost twice $C / A$. It is shown that the controller proposed in this paper is still capable to regulate the desire position asymptotically.

\subsection{Test Rig}

Figure 2 shows the schematic of the hydraulic test rig. The hydraulic circuit consists of an actuator controlled by a Moog D765 high-performance servovalve, mounted on a reinforced steel table. The actuator has an annulus area of $0.98 \mathrm{in}^{2}$ and a $24 \mathrm{in}$ stroke. The servovalve can flow 34 $\mathrm{L} / \mathrm{min}$ at $3000 \mathrm{psi}$ and has a rise time of $2 \mathrm{~ms}$. It uses a mechanical feedback spring with a linear variable differential transformer (LVDT) that measures the position of the spool. Two Sensotech-FPG transducers measure the 
fluid pressure at the actuator chambers. A rotary encoder with a resolution of 1024 counts/revolution (linear resolution of $0.0015 \mathrm{in}$ ) establishes the relative position of the actuator. The data acquisition system is comprised of a personal computer and a DAS-16F input/output board.

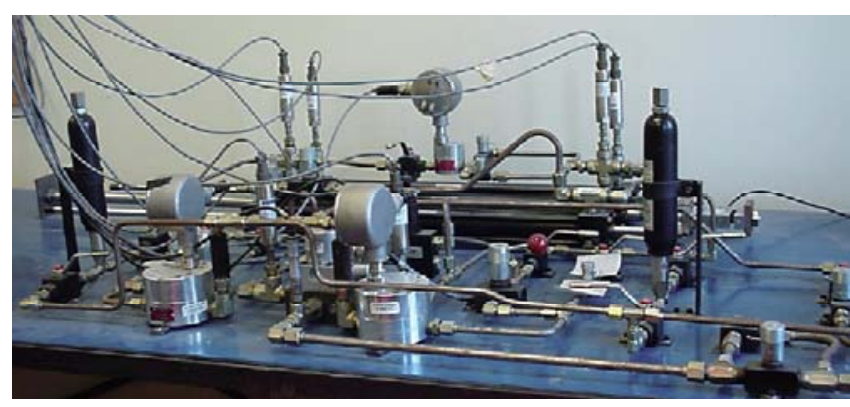

Fig. 2 Schematic of the experimental test rig.

\subsection{Results}

In all experiments, the actuator accelerated from the rest to the desired position $x_{d e s}=8$ in under controller (7). The first set of experiments was performed using the ratio of the control gains $K_{p} / K_{x} \cong C / A$ which is the limit of stability region for the control system (see Section 5). The hydraulic compliance of the test rig was over-estimated as $C=7.14 \times 10^{-5} \mathrm{in}^{3} / \mathrm{psi}$. Knowing $A=0.98 \mathrm{in}^{2}$, the control gains were chosen to be $K_{x}=0.1 \mathrm{~V} / \mathrm{psi} i^{1 / 2}$ in and $K_{p}=8 \times 10^{-6}$ $V / p s i^{3 / 2}$ which do not saturate the actuator at the extreme measurable limits of motion with the supply pressure equal to $P_{s}=2000$ psi.

Figure 3 shows the response of the system. The position response of the system demonstrates the convergence of the

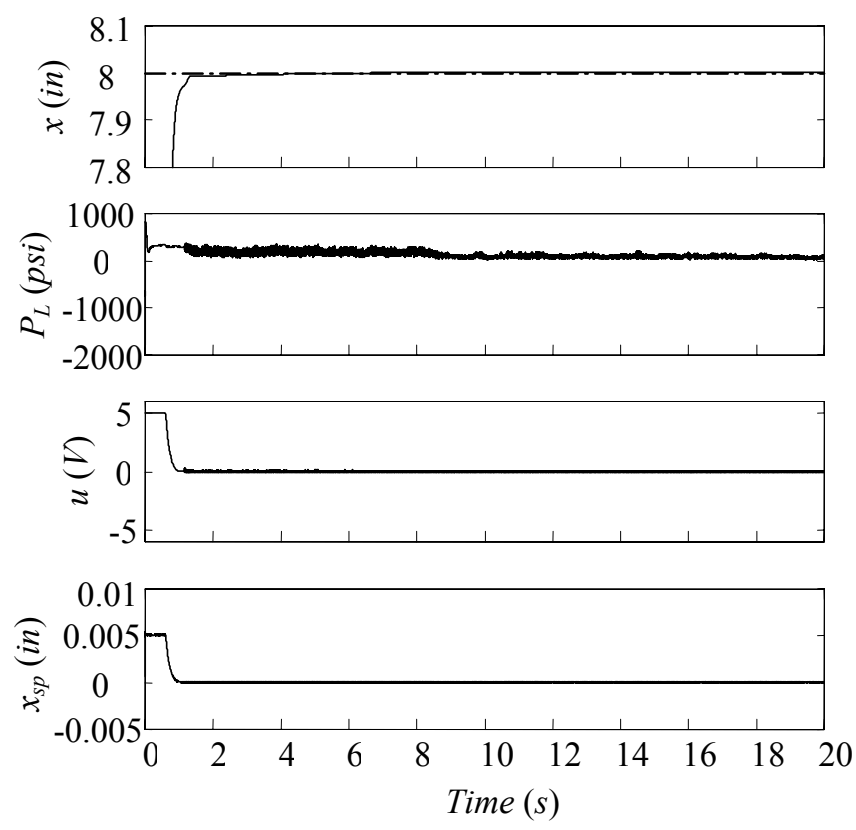

Fig. 3 System response; $K_{x}=0.1 \mathrm{~V} / \mathrm{psi} i^{1 / 2} i n$ and

$$
K_{p}=8 \times 10^{-6} \mathrm{~V} / \mathrm{psi}^{3 / 2} \text {. }
$$

system trajectory to the desired position with no steady state error and verifies the friction compensation capability of the proposed controller in a hydraulic actuator. Note that the position steady state error in Fig. 3 is observed to be 0.0014 in which is within the resolution of measurements and cannot be detected by the controller. Knowing the experimentally derived value of the maximum static friction, $F_{S}=314.72 \mathrm{lb}$, Fig. 3 also confirms that the steadystate supply pressure is within the range denoted in (11).

In order to observe the performance of the control scheme when the control gain ratio is greater than $C / A$, the second set of experiments with the same position regulation task was setup with $K_{p} / K_{x} \cong 2 C / A$. Increasing the gain ratio was done by either increasing $K_{p}$ or decreasing $K_{x}$. Figure 4 illustrates the system response in the first case. It is shown that the proposed control system convergences to the desired position with no steady state error. Comparing this response (solid line) with the response of the system with $K_{p} / K_{x} \cong C / A$ (dashed line) reveals that decreasing $K_{x}$ results in a slower response with larger rise time.
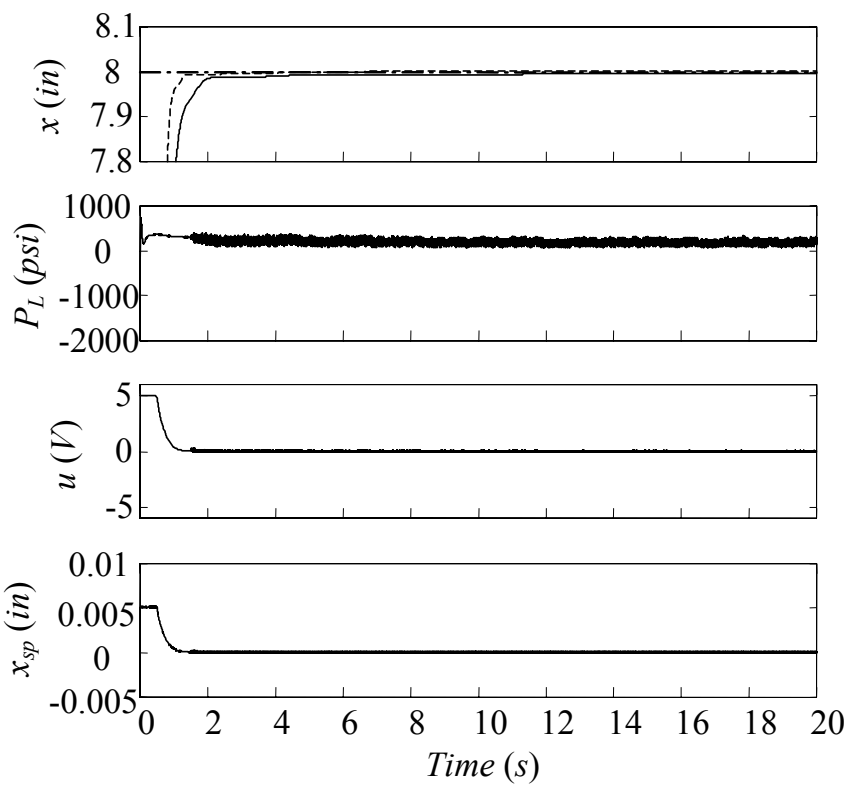

Fig.4 System response; $K_{x}=0.05 \mathrm{~V} / \mathrm{psi}^{1 / 2}$ in and

$$
K_{p}=8 \times 10^{-6} \mathrm{~V} / \mathrm{psi}^{3 / 2} \text {. }
$$

The gain ratio can also be doubled by doubling $K_{p}$ and keeping $K_{x}$ unchanged. Figure 5 shows the system response. It illustrates that although increasing $K_{p}$ dampens the system response (the position response of the system with $K_{p} / K_{x} \cong C / A$ is shown by dotted line), the response is still asymptotically converging to the desired position without steady state error.

\section{Conclusions}

Extension of Lyapunov direct method to nonsmooth systems was employed to design a friction compensating 


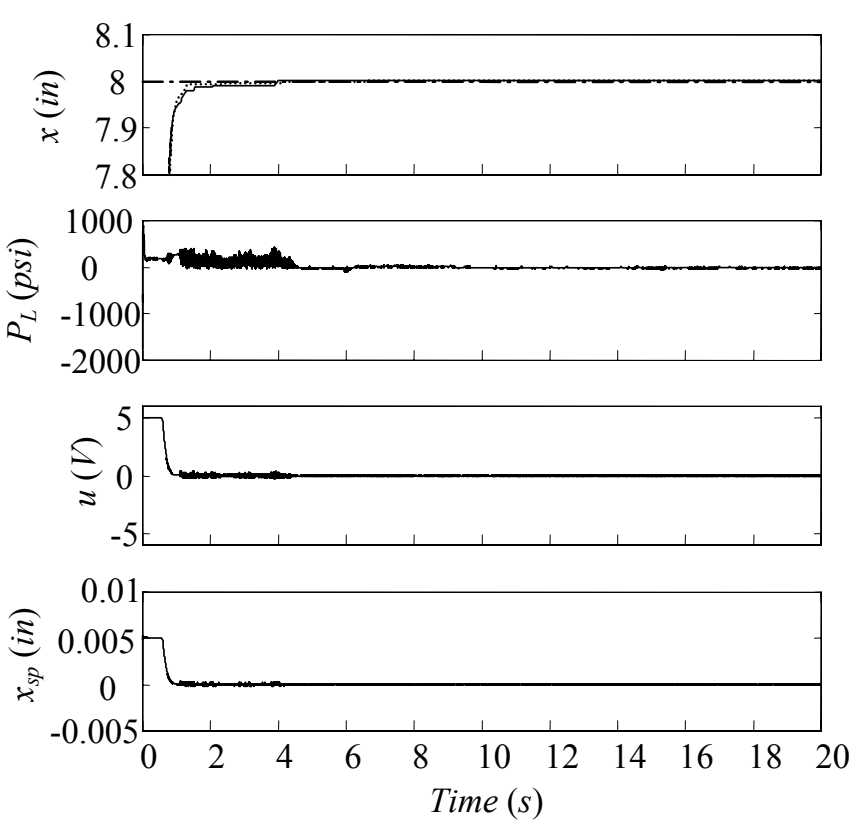

Fig.5 System response; $K_{x}=0.1 \mathrm{~V} / \mathrm{psi}^{1 / 2}$ in and

$$
K_{p}=16 \times 10^{-6} \mathrm{~V} / p s i^{3 / 2} \text {. }
$$

position controller for hydraulic actuators. The control scheme is capable of asymptotic position regulation with no steady-state error in spite of friction effects. Although no knowledge of the friction model, servovalve dynamics, or hydraulic parameters is required for control action, stability and effectiveness of the control scheme in the presence of all these parameters is verified both analytically and experimentally. Due to the discontinuity of the control law and the friction model, the existence, continuation and uniqueness of the solution were, first, proven using Filippov solution theories. The extension of LaSalle's invariance principle to nonsmooth systems was then employed to prove that all the solution trajectories converge to the equilibria. Experiments conducted on a test bed verified the effectiveness of the proposed controller in position regulation in the presence of actuator friction.

\section{References}

[1] Vossoughi, G., and Donath, M., "Dynamic feedback linearization for electrohydraulically actuated control systems", ASME J. Dyn. Systems, Meas Control, v 117, pp 468-477, 1995.

[2] Yao, B., Bu, F., Reedy, J., and Chiu, G., "Adaptive robust motion control of single-rod hydraulic actuators: Theory and experiments", IEEE/ASME Trans. Mechatronics, v 5, n 1, pp 79-91, 2000.

[3] Bonchis, A., Corke, P. I., and Rye, D. C., "Experimental evaluation of position control methods for hydraulic systems", IEEE Tran. Control Systems Technology, v 10, n 6, pp 876-882, 2002.

[4] Sirouspour, M. R., and Salcudean, S. E., "Nonlinear control of hydraulic robots", IEEE Trans. Robotics and Automation, v 17, n 2, pp 173-182, 2001.
[5] Tafazoli, S., de Silva, C. W., and Lawrence, P. D., "Tracking control of an electrohydraulic manipulator in the presence of friction", IEEE Trans. Control Systems Technology, v 6, n 3, pp 401-411, 1998.

[6] Lischinsky, P., Canudas de Wit, C., and Morel, G. "Friction compensation for an industrial hydraulic robot", IEEE Control Systems Magazine, v 19, n 1, pp 25-32, 1999.

[7] Hwang, C. L., and Lan, C. H., "The position control of electrohydraulic servomechanism via a novel variable structure control", Mechatronics, v 4, n 4, pp 369-391, 1994.

[8] Niksefat, N., Wu, C. Q., and Sepehri, N., "Design of a Lyapunov controller for an electro-hydraulic actuator during contact tasks", ASME J. Dyn. Syst. Measurement Control, v 123, pp 299-307, 2001.

[9] Sohl, G. A., and Bobrow, J. E., "Experiments and simulations on the nonlinear control of a hydraulic servosystem", IEEE Trans. Control Systems Technology, v 7, n 2, pp 238-247, 1999.

[10] Canudas de Wit, C., Noel, P., Aubin, A., and Brogliato, B., "Adaptive friction compensation in robot manipulators. Low velocities", Int. J. Robotics Research, v 10, n 3, pp 189-199, 1991.

[11] Armstrong-Helouvry, B., Dupont, P., and Canudas de Wit, C., "A survey of models, analysis tools and compensation methods for the control of machines with friction", Automatica, v 30, n 7, pp 1083-1138, 1994.

[12] Southward, S. C., Radcliffe, C. J., and MacCluer, C. R., "Robust nonlinear stick-slip friction compensation", ASME J. Dyn. Systems Meas. Control, v 113, pp 639-645, 1991.

[13] Filippov, A. F., "Differential equations with discontinuous right-hand side", Math. Sbornik 51, 99, 1960 (English Translation: American Math. Society Translations v 42, pp199-231, 1964).

[14] Filippov, A. F., "Differential equations with second members discontinuous on intersecting surfaces", Differentsial'nye Uravneniya 15, 1814, 1979 (English Translation: Differential Equations v 15, pp 1292-1299, 1980).

[15] Shevitz, D., and Paden, B. "Lyapunov stability theory of nonsmooth systems", IEEE Transactions on automatic control v 39, n 9, pp 1910-1914, 1994.

[16] Wu, Q., Onyshko, S., Sepehri, N. and ThorntonTrump, A.B., "On Construction of smooth Lyapunov functions for non-smooth systems", International Journal of Control, v 69, pp 443-457, 1998.

[17] Merritt, H. E., Hydraulic Control Systems, John Wiley, NY, 1967.

[18] Alleyne, A., "Nonlinear force control of an electrohydraulic actuator", Japan/USA Symposium on Flexible Auto., Boston, MA, pp 193-200, 1996. 